\title{
RANK PRESERVATION AND RANK STRUCTURE OF JUDGEMENT MATRIX
}

\author{
MANG NING THE BAN GUANG VEN - \\ Huazhong University of Science and Techonolgy \\ Muhne, P.R. China
}

ABSTRACT

This paper is written for the rank preservation of evaluating priority weigts and the rank structurein a positive reciprocal or approxinate positive reciprocal natrix $A$ that is inconsistent. It witl be shown that the satisfying rank structrue of the metrix is an important condition to preserve rank of solutions. Therefore, It is better to first wo had better tost the rank correlation for tho rank structure antrix and second evaluate the priority weights. Besides the rank corretation of uatrix $A$ can be checked easily.

\section{INTRODUCTION}

We know that many social variables are unzeasurable in soci-systez analysis. In order to estimate ther elative priority weights of these variables or objects, wany evaluating methods have been advanced. Since systezatic variabies and objects are unneasurable, accuracy is nat the most impotant factor, however the priority rank orderwill becose the basic and stable retationship in the systea analysis. Therefore, T.L. Saty [4] states that for an inconsistent watrix $A$, rank preservation is ore rriterion to evaluate which of the priority weight aethods is best. and he have shown that the eigenvector aethod (EM)(Saaty, 1977,1980) is an asymptotic preserving rank wethod.

Usually, the judgement antrix $A=\left(a_{i j}\right)$ can be in following situation, 1. Evey tine the pariwise comparison judgement enabte to produce the same informtion about judge's preference as possible.

2. The jugement matrix strives.towards positive reciprocal, but it is not positive reciprocal perhaps.

3. If overy judgement is independent and thereis no effect between objects, then there aust exist no transmission in priority weights.

THE GEONETRIC DESCRIPTION OF THE JUGENENT MATRIX

Let us assuse that the rows of atrix $A=(a)$ are eleaents of the vector space $R_{n}^{+}$, where $\quad R_{1}^{t}=\left(\left(a_{n,}, a_{i,}, \ldots, a_{n}\right): a_{1 j}>0, i, j=1,2, \ldots, n\right)$.

and. the weight voctor subspace $x_{n-1}^{*}$.

where $\quad x_{n-4}^{*}=\left\{\left(w_{1}, x_{2}, \ldots, w_{n}\right) t\left(x_{k}\right) \in R_{n}^{*}, \sum_{k=1}^{n} w_{k}=1 \mid\right.$

There exists the an $R_{\mathrm{n}} \rightarrow \mathrm{W}_{\mathrm{n}-1}^{4}$.

we have $\quad w_{k}=1 \cdot a_{i x} \sum_{i=1}^{n} t_{i k} \quad k=1,2, \ldots, n$.

Sinilarly, assume that the coluans of matrix A are elements of the $R_{i}^{+}$, for the aap $R^{t}$ $\rightarrow w_{n}^{n}-1$, we have

$$
n_{k}=1 \cdot \sum_{i=1}^{n} a_{i x} \quad k=1.2 . \quad n
$$

In the following, we take the rows to be the discussion object, and all analysis restuts are appliance to the coluans Let a be the ith row vector According to the 
formula (1), $\alpha^{\prime} a_{i} \in R_{n}^{+}$can be considered an element for the weight vector, where $\forall d^{\prime} \in R_{s}^{*}$, so that we project $R^{t}$ on the hypersurfase $S_{n-1}^{+}$.

where $\quad S_{n-1}^{+}=\left\{\left(a_{1 p}, \ldots, z_{t}\right) \mid\left(a_{1, i}\right) \in R^{+}, \quad\{0 ; 1=1\}\right.$,

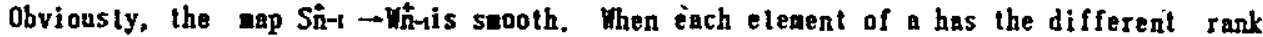
degree, which is called the unequal priority rank row, the rank vector of a is denoted $\bar{x}_{1}=(1,2, \ldots, n)$; when there exist the sane rank degree etenents in the row, we denote the rank vector of ai to $\bar{b}_{i}=(1,1.5,1.5,4, \ldots, n)$, for example.

DEFINTION 1. Iwo row vector $a_{i}$. $a_{j} \in$ Sth-thave the relation of the saze rank order if for each $k, t=1,2, \ldots, n, a_{i k}>a_{j l}$ and $a_{j k}>a_{j L}$; denote $a_{i j} E a_{j}$.

THEOREN 1. Equivalence relation ER deternines a uniqe classification $S_{2}^{+}-1$ /ER .

PROOF, The sare rant retation ER is a rank equivalence relation since ER is a tro-ary relation, and (1) if $a_{i} \in S^{*}-1$, then $a_{i} E R a_{j} ;$ (2) if $\forall a_{i}, a_{k} \in S_{n-1}^{*}$ and $a_{i} E R a_{k}$, then $a_{k} E R a_{i} ;$ (3) if $\forall a_{i}, a_{x}, a_{j} \in S_{n-1}^{+}, a_{i} E R a_{k}$ and $a_{k} E R a_{j}$, then $a_{i} E R a_{j}$. Certainly, $\forall a_{0}$ $\in S_{n}^{*}-1$ nust be in an equivalence class at least, for example $\vec{s}_{0}=\left(a_{i} \in S_{n}^{+} \rightarrow 1\right.$ a; ER a. $f$, thus $S_{n}^{+}-1=\left\lfloor\left(\bar{s}_{i}: a_{i} \in S_{n-1}^{+}\right)\right.$. On the other hand, suppose two classes be satisfied $\bar{s}_{i} \eta_{i} \bar{s}_{j} \neq 0$, thus at least exsits one vector $a \in \bar{s}_{1}$ and $a \in \bar{s}_{2}$, for $\forall a_{1} \in \bar{s}_{1}, \exists a_{2} \in \bar{s}_{2}$, a, ERa and $a_{2} E R a$, so that $a_{1} E R a_{2}$, that is $\bar{s}_{1} \in \bar{s}_{2}$. Sinitarly, we show $\bar{s}_{2}<\bar{s}_{1}$, therefore $\bar{s}_{1}=\bar{\zeta}_{2}$. The uniqueness of ctassification is obvious.

THEOREN 2. On the hypersurface $S_{n-1}^{+}$there exist $\mathrm{N} !$ rank equivalence ctasses in which all elenents are unequal priority rank rows.

PROOF, In general, let one of these rank equivalence $c$ lasses be denoted $\bar{\tau}=(1,2, \ldots, n)$, the number of this classes is equal to the number of naps of itself. So that "1" has $n$ images of the map, "2" has (n-1) images, " $3 "$ has (n-2) images, and so on. Therefore, the number of these classes is $\mathrm{N}$ ! .

Now consider the rank geometric structure of the antrix $A$. The unequal priority rank classes as stated above can be destribed the (n-1)-din hyperfaces. It is not difficult to iangin that the classes whose dimensian are $<(n-1)$ are between the (n-1)-dir classes, which are called the bounary rank equivalence classes. See Figure 1 wich shows $n=3$. In order to represent the rank correlation degree of two row rank vectors, we define the vectorial angle to be the norm. It ought to be noted that if the angle is equal to zero, then the rank order of two row vectors, nust be perfectly correlative: but rows have perfect rank corretation, the their angles mast not be zero.

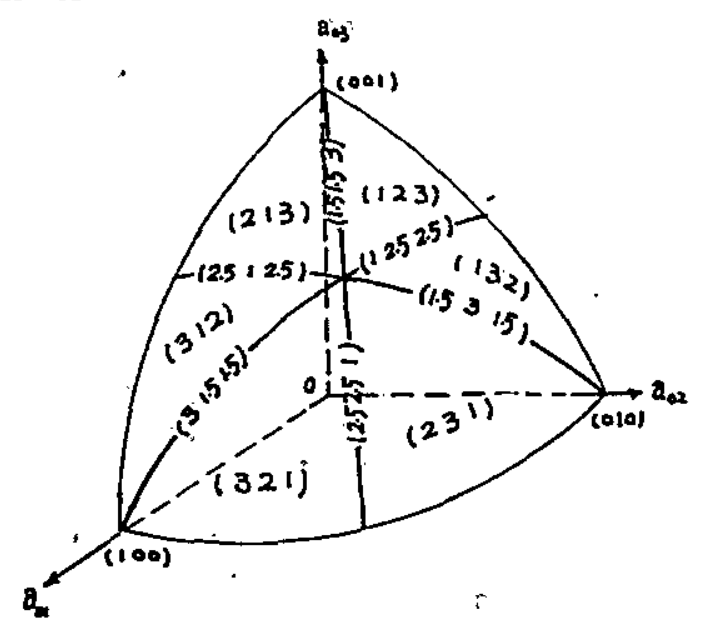

(Figure 1) 
Conparison OF EVALUTING NETHODS

Many evaluting aethods have been pat foward as foltows,

the eigenvector mothod (EM). $\quad A \cdot Y=\lambda$ max:W ,

where $\lambda_{\text {mas }}$ is the principal eigenvalue of the jugenent iatrix $A_{;}$.

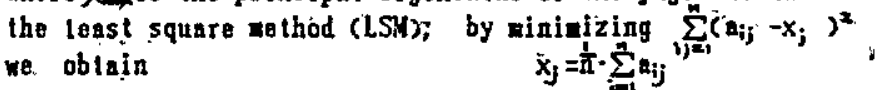

$\check{x}_{j}=\frac{1}{\pi} \cdot \sum_{i=1}^{n} a_{i j} \sum_{j=1}\left(a_{i j}-x_{j}, j=1,2, \ldots, n\right.$;

the togarithric teast square wethod (LSW), by mininizing $\sum_{j=1}^{n}\left(\log a_{i j}-\log x_{j}\right)^{z}$,

we obtain $\quad x_{j}=\left(\prod_{1}\left\{a_{1\}}\right)\right.$

$\mathrm{j}=1,2, \ldots, \mathrm{n}$;

where $\left(x_{2}\right) \in R_{4}^{+}$. Normalized by $(1)$, LSH and LLSW yield the priority woights. Others, the nornalization of the geonetric of the rows (NGK) [3], the noraalization of the cotunn and sum of the rows (NCM) [1], ect.. It is natural that the priority reights evaluated by these aethods not be equal in general.

Consider the following $3 \times 3$ judgenent antrix

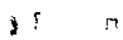

$$
\therefore \quad-\quad \begin{gathered}
A^{\prime} \\
\therefore
\end{gathered} \quad\left(\begin{array}{ccc}
1 & 4.1 & 1 \\
1 / 4 & 1 & 11 \\
1 & 4.1 & 1
\end{array}\right)
$$

See Figure 1 , all rows are in one rank equivalunce class (1.5. 3, 1.5 ), Ye can directly estinate that: the rank of the weight múst be $(2.5,1,2.5$ 2, that is

$w_{A^{\prime}}=w_{i}>w_{B^{\prime}}$. Applying each method as above, we have;
EM
NGK
$\mathrm{NCK}$
LSN
LLSM
A' 0.4448
0. 4448
0.4448
$0 . \$ 455$
Q. 14453
B' 0.1103
0.1103
0.1103
0.1090
0.1090
D. 4448
0.4448
0.4448
0. 4455
$0.445 \lambda^{\circ} \quad \lambda_{\max } \approx 3.0166$

Let us take a symetric perturbation on the rank class(1.5, 3, 1.5), for example, it produce the following artrix,

$$
\begin{aligned}
& A^{\prime} \\
& \text { B }^{\prime}
\end{aligned}\left(\begin{array}{ccc}
1 & 4 & 1.1 \\
1,4 & 1 & 1,4 \\
1.1 & 1 & 1
\end{array}\right)
$$

Though rows of this aatrix are not in one rank class, the aatrix has the saar row rank order and the same column rank order. Similarly, we can estuate. that the rank order of weights is $(2.5,1,2.5)$, that is $w_{A}=w_{C}>w_{B^{\prime}}$, solutions as followis

$\begin{array}{llllllll} & \text { EN } & \text { NGN } & \text { NCM } & \text { ISN } & \text { LLSM } & & \\ A^{\prime} & 0.4461 & 0.4460 & 0.4460 & 0.1423 & 0.4429 & \\ B^{\prime} & 0.7079 & 0.1080 & 0.1080 & 0.1155 & 0.1143 \\ C^{\prime} & 0.4461 & 0.4460 & 0.4460 & 0.4423 & 0.4429 & \lambda_{\max } \approx 3.0674 & \end{array}$

With a unsymetric perturbation, we obtain the matrix, for example,

$$
\text { B' }^{\prime}\left(\begin{array}{ccc}
1 & 1 & 1.1 \\
0.26 & 1 & 0.25 \\
1.1 & 1 & 1
\end{array}\right)
$$

\begin{tabular}{|c|c|c|c|c|c|c|}
\hline EX & $N G M$ & - NCH & LSN & LLSN- & & \\
\hline 0.4454 & 0.4454 & 0.4154 & -0.4412 & 0,4396 . & $3^{n}$ & 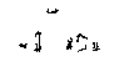 \\
\hline $\begin{array}{l}0.1052 \\
0.4454\end{array}$ & $\begin{array}{l}0.1092 \\
\text { B. } 1454\end{array}$ & $\begin{array}{l}0.1092 \\
0.4452\end{array}$ & $\begin{array}{l}0.1157 \\
0.4433^{*}\end{array}$ & $\begin{array}{l}031150^{1} \\
0.4453\end{array}$ & $\lambda_{\operatorname{mx}} \approx$ & :- \\
\hline
\end{tabular}

At first, we shall estimate that the sotutions of EN, NGM and NCY may be chang a tiltte and however the solutions of LSM and LLSN have taken great changed obviosty, as such, 
Coxpare above results, and we note that for a perturbation a variety of aethods represent different degrees on sensitivity or stability. It is natural to ask which sotutions of aethods represent judge's information and which aethods can preserve rank?

DEFINITION 2. A method of solution is said to preserve rank if $a_{i k}>a_{i v}$ for $i=1,2, \ldots . n$, it yietds $x_{k}<w_{L}$; a nethod is said to aymptotical preserve rank if as $n \rightarrow \infty$, it holds above situation.

THEOREM 3. EM, NGN, NCK, LSM and LLSM preserve rank (Saaty, 1984).

PROOF, For a positive reciprocal of approxinate positive reciprocal matrix that is inconsistent, froa $a_{i k}>a_{i k} i=1,2, \ldots, n$, we have $a_{x j}<a_{i j} j=1,2, \ldots, n$.

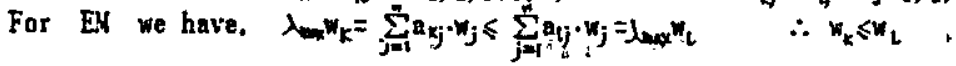

For NGM, $\because \overline{\bar{m}}_{k}=\left(\prod_{j=1}^{n} a_{k j}\right)^{\frac{1}{n}} \leqslant\left(\prod_{j=1}^{h} a_{j}\right)^{\frac{1}{n}}=\overline{\bar{w}}_{i}$

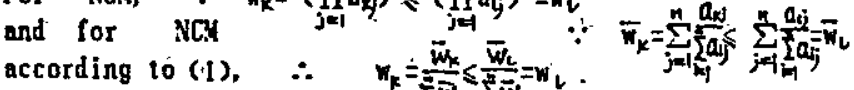

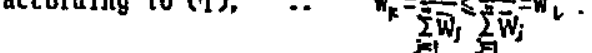

For LSK and LLSN, directly by $a_{i k}>a_{i l}, i=1,2, \ldots, n$,

0

LSN has $x_{k}=\frac{1}{n} \cdot \sum_{i=1}^{n} a_{k} \geqslant n_{n}^{1} \cdot \sum_{i=1}^{n} a_{i l}=x_{l}, \therefore x_{k} \leqslant n_{i}$,

and LLSW has $\quad x_{k}=\left(\prod_{i=1}^{n} a_{k}\right)^{\hbar} \geqslant\left(\prod_{i=1}^{n} a_{i}\right)^{k}=x_{L} . \quad \therefore w_{k} \leqslant w_{i}$.

COROLLARY. If all rows in one rank equivalence class, then EN, LSM, LLSM, etr. preserve rank.

PROOF, Since ali rows have the same rank order, for $i=1,2, \ldots, n$, we have $a_{i k}>a_{i l}$ So that is obsvious by Theoren 3.

Me now develop above result for rank preservation.

DEFINITION 3. If two rark classes $\bar{r}_{i}$ and $\bar{r}_{j}$ have the angle $\angle\left(\bar{r}_{j} \bar{r}_{j}\right)=0$, as $n \rightarrow x$, we say that the rank orders of these classes are perfectly corretative asyaptotically.

THEOREN 4. Arbitrary adjacent rank equivalunce classes are perfectly rank correlative asymptotically.

PROOF, In order to prove this resutt we introduce two teras of the perautation group, comatation and circulantion. Generally we denote one $(n-i) d i r$ rank $c(a s s ~ i=(1,2, \ldots, n)$, define the adjacent connutation,

define the adjacent circulation,

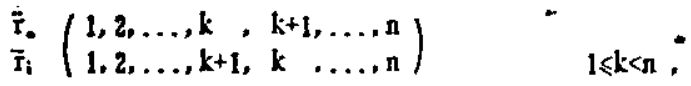

$$
\overline{\bar{r}}_{3}\left(\begin{array}{l}
1,2,3, \ldots, n \\
n, 1,2, \ldots, n-1
\end{array}\right) \text { or }\left(\begin{array}{ccc}
1,2, \ldots, n-1, n \\
2,3, \ldots & . n & .1
\end{array}\right) \text {. }
$$

(1) For the adjacent comsutation, let the angte of $\bar{r}_{\text {. }}$ and $\bar{r}_{i}$ be $a=-\left(\bar{r}_{,} \bar{r}_{i}\right)$, we have

$$
\cos a=\frac{1+2 \cdot 2+\cdots+k(k+1)+(k+1) k \cdot \cdots+n \cdot n}{1^{2}+2^{2}+\cdots+n^{2}}=\frac{6}{(n+1)(2 n+1) n}
$$

as $n=9, \cos \alpha=0.9985 \quad \alpha ; 4.8 ; \quad$ as $n \rightarrow x$, then $\cos \alpha=1, \quad \therefore \quad a \rightarrow 0$.

and in case of adjacent circulanting iransforating, fet $\beta=L\left(\vec{r}_{,} \bar{r}\right)$, we have

$$
\cos \beta=\frac{1 \cdot n+2 \cdot 1+3 \cdot 2+\cdots+n(n-1)}{1^{2}+2^{2}+\cdots \cdot n^{2}}=1-\frac{3(n-1)}{(n-1)(2 n-1)}
$$

as $n=9, \cos \beta=0.8737, \therefore \beta=29.1$; as $n \rightarrow x$, then $\cos \beta=1 . \therefore \beta \rightarrow 0$ 
(2) Consider the further raijk "classas fron $r$, which bo produced by the k-circulation of $\bar{r}_{*}, \quad \bar{r}_{0}\left(\begin{array}{l}1,2, \ldots, k, k+1, k+2, \ldots, n \\ n-k+1, n-k+2, \ldots, n, 1,2, \ldots, n-k\end{array}\right)$

where $1 \leqslant k<n$, when $k=1$, that is an odjacent circulation, let $\quad y=\angle\left(\bar{x}_{*} \bar{x}_{v}\right)$

$$
\cos \gamma=\frac{1(n-k+1)+2(n-k+2)+\ldots+k n+(k+1) 1+\ldots n(n-k)}{1^{2}+2^{2}+\ldots+n^{2}}=1-\frac{3 k(n-k)}{(n+1)(2 n+1)}
$$

If and only if $\mathrm{k}$ is finite, then as $n \rightarrow \infty, \cos =1 . \quad \therefore y \rightarrow 0$. Obsviously the bounnry rank equivalence classes that are lotween $\bar{r}_{\text {. }}$ and $\bar{r}_{i}$ or $\bar{r}_{\text {. }}$ and $\bar{r}_{j}$ will be perfectly rank corralative asymptotically, 100.

THEORE 5. If the rows of the jugenent antrix are in the adjacent or near rank classes, EN, NGN, NCK, LSK and LLSN all preserve rank asymptoticatly.

PROOE, According to Theorew 4, becuase two arbitrary rows aro rank correlative asymptotically, there exists $B 0$, as $n \geqslant N$, we have $a_{i k}>a_{i h}$ for $i=1,2, \ldots, n$. So that is evident from Theoren 3.

Sumarizing as above, we have shown that the property of rank preservation is of the rank structure of eatrixes. Therefore, consider the relation of the rank geonetric structure of the entrix A to the gethods of EM. NGM. NCY, LSN, LLSM, etc.. we have following two results,

1. If all rows of the matrix $A$ is in a rank equivalence class, then the evaluating method as above can be said to preserve rank.

2. If the rows of the matrix $A$ is in some adjacent rank equivalence classes then the evaluating method of solution preserves rank asymptotically.

\section{PROCEDURE OF COKPUIATION}

It is clear that the satisfactory rank structure of metrix $A$ is important condition to reserve rank of solutions. At first we had better test the rank correlation of the aatrix and adjust the rank structure by consulting with the judge, and second evaluate the priority weights. By this way, ne will obtain following advances;

(1) The useful information about judge's favor could be retained by consulting.

(2) The rank preservation of evaluating could be held up.

(3) It could evade recalculation.

Now let introduct Kendall's rank correlation coefficient be the rank corrlation index of the matrix $A$, here

$$
\tau=\frac{\sum\left(R j-1 \cdot n \cdot \sum_{i n j}^{n} R j\right)}{1 / 12 \cdot n^{3}\left(n^{2}-1\right)} \quad \text { o } \tau \leqslant 1
$$

where $\mathrm{Rj}$ is the sus of the elements in $\mathrm{j}$ th column. How large is the satisfactory index value $\tau$ ? we assume that the domain of the rank adjacent rows determine the satisfactory corretation index value $\tau_{4}$, as following aatrix described. See Figure $l$, in this natrix one row can be produced from one another by commatating.

by the formula (3).

$$
\left(\begin{array}{ccccccc}
1 & 2 & 3 & 1 & \ldots & n-1 & n \\
2 & 1 & 3 & 4 & \ldots & n-1 & n \\
1 & 3 & 2 & 1 & . & n-1 & n \\
\cdot & * & & \cdot & \ldots & \cdot & \cdot \\
1 & 2 & \ddots & \vdots & & \cdot & \cdot \\
1 & 2 & 3 & 4 & \ldots & n & n-1
\end{array}\right)
$$




$$
\tau_{d}=\frac{2\left(n^{2}-n-1\right)}{1 \cdot 12 n^{3}\left(n^{2}-1\right)}
$$

Let us note that in comparison with the randox index (RI), $\tau_{j}$ is correspond to RI on $S^{+}-1$

The following table gives the order of the matrix (first row) and the rank satisfaction index value $\tau_{1}$,

$$
\begin{array}{lllllllll}
n_{1} & 2 & 3 & 4 & 5 & 6 & 7 & 8 & 9 \\
\tau_{3} & 0 & 0.44 & 0.73 & 0.65 & 0.91 & 0.94 & 0.96 & 0.97
\end{array}
$$

Here we give one example considered the following $4 \times 4$ judgenent atrix, whose row rank structure matrix is on the right,

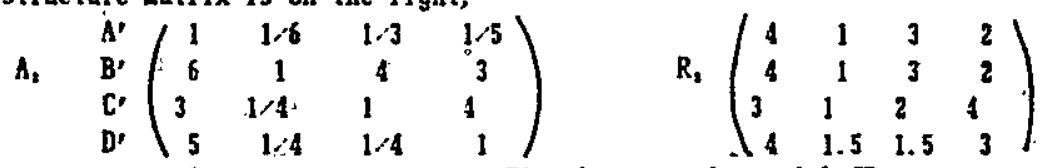

By (3), we can easily get $\tau=0.23<0.73=\tau_{4}$, in comparison with $C R=0.158>0.10$ [2]

So we ought to consult with judges and adjust the matrix. Assure the new aatrix as follows,

$$
A_{1} \quad \begin{aligned}
& B^{\prime} \\
& C^{\prime}
\end{aligned} \quad\left(\begin{array}{cccc}
1 & 1 / 6 & 1 / 3 & 1 / 5 \\
6 & 1 & 4 & 3 \\
3 & 1 / 4 & 1 & 1 \\
5 & 1,3 & 1 & 1
\end{array}\right) \quad R, \quad\left(\begin{array}{cccc}
4 & 1 & 3 & 2 \\
4 & 1 & 3 & 2 \\
4 & 1 & 2.5 & 2.5 \\
4 & 1 & 2.5 & 2.5
\end{array}\right)
$$

\begin{tabular}{|c|c|c|c|c|}
\hline EN & NGM & NCN & LSH & LLSM \\
\hline 0.0619 & D. $B 612$ & 0.0633 & 0.0675 & 0.0612 \\
\hline 0.5502 & 0.5492 & 0.5450 & 0.5782 & 0.5492 \\
\hline 0.1733 & 0.1754 & 0.1733 & 0.1598 & 0.1754 \\
\hline 0.2146 & 0.2142 & 0.2185 & 0.1946 & 0.2142 \\
\hline
\end{tabular}

Thus we have $\tau=0.925>\tau_{4}$, comparing with $C R=0.039<0.10$. Now let us estinate and obtain solutions,

8

1. Zhuo Huar Chen, $X_{u}$ Shu Bai, He Jin Sheng, "The Anelytic Hierarchy Process", Science Press, Beijing, P.R. Chiza, 1986.

2. T.L.Saaty," The Analytic Hierarchy Process ", MeGraw Hill, Inc. 1980.

3. T.L.Saaty, L.G.Vargas, "The Logic of Prioritles ", Boston, Kluwer. Nijhoff Publishing, 1982.

4. T.L.Saaty, L.G. Vargas, "Inconsistengy and Rank Preservation", Journat of Matnrmaticat Psychology, vol.28, No. 2, 1984.

5. S. Sieget, "Nonparaxetic Statistics for the Behavioral Sciences ", MeGran Hill Book Coupany. New York, 1956. 